\title{
Investigating $\beta$-lactam drug targets in Mycobacterium tuberculosis using chemical probes
}

\author{
Author List: Samantha R. Levine and Kimberly E. Beatty ${ }^{\star}$ \\ *beattyk@ohsu.edu \\ Program in Chemical Biology and the Department of Biomedical Engineering \\ Oregon Health \& Science University, Portland, Oregon 97201, United States
}

\begin{abstract}
Tuberculosis is a deadly disease that requires a flexible arsenal of drugs to treat it. Although $\beta$-lactam antibiotics are rarely used to treat Mycobacterium tuberculosis (Mtb) infections today, the targets of these drugs are present in the bacterium. Moreover, the cell wall peptidoglycan of Mtb contains an abundance of unusual $(3 \rightarrow 3)$ cross-links. These cross-links are formed by enzymes called L,Dtranspeptidases, which are susceptible to inhibition by the carbapenem class of antibiotics. We developed new small molecule probes to investigate the L,D-transpeptidases and other $\beta$-lactam drug targets in Mtb. We synthesized probes based on three classes of antibiotics, a monobactam, cephalosporin, and carbapenem. For the carbapenem, we synthesized a meropenem analogue conjugated to a far-red fluorophore. This probe was particularly useful in identifying active L,Dtranspeptidases in protein gel-resolved lysates. Next we analyzed $\beta$-lactam targets in lysates from both hypoxic and actively-replicating cultures of $M t b$. We identified numerous targets, including transpeptidases, carboxypeptidases, and the $\beta$-lactamase BlaC. Overall, we provide evidence that $M t b$ dynamically regulates the enzymes responsible for maintaining cell wall peptidoglycan and that meropenem is a good inhibitor of those enzymes.
\end{abstract}

\section{Introduction}

Tuberculosis (TB) is the most deadly infectious disease in human history. Over time, Mycobacterium tuberculosis (Mtb) strains have developed resistances to all anti-mycobacterial drugs. Drug-resistant strains occur worldwide and cause $\sim 6 \%$ of TB infections ${ }^{(1)}$. The global rise of drug resistance necessitates finding new drugs, a slow and expensive process, or repurposing existing drugs $^{(2-4)}$. New TB treatments would ideally target both active TB and latent TB infections (LTBI). In the human host, Mtb encounters environmental stresses that can trigger a dormant, non-replicating state. These dormant bacteria exhibit phenotypic drug resistance to most front-line drugs ${ }^{(5-7)}$. While dormant $M t b$ is associated with LTBIs, active TB also presents with metabolically diverse populations ${ }^{(5-7)}$. There is an urgent need to identify and validate therapeutics that will be broadly useful for the majority of TB patients.

$\beta$-lactam antibiotics are widely used drugs that target cell wall biosynthesis. Two of the earliest classes of $\beta$-lactams, the penicillins and cephalosporins, inhibit D,D-transpeptidases. These enzymes are penicillin-binding proteins (PBPs) that modify cell wall peptidoglycan. They form $4 \rightarrow 3$ cross-links between a stem peptide's fourth amino acid, D-Alanine, and the third amino acid on an adjacent stem: meso-diaminopimelic acid (mDAP) ${ }^{35,36}$. In most bacteria, the peptidoglycan is comprised of $4 \rightarrow 3$ linkages. However, in $M t b 3 \rightarrow 3$ cross-links predominate and comprise up to $80 \%$ of linkages ${ }^{(8,9)}$. This covalent modification, from $\mathrm{mDAP}^{3}$ to $\mathrm{mDAP}^{3}$, is generated by L,D-transpeptidases (LDTs). A recent 
review describes the structure and function of Mtb's PBPs and LDTs ${ }^{(10)}$. Notably, Ldt $_{\mathrm{Mt1}}$ and $\mathrm{Ldt}_{\mathrm{Mt} 2}$ are essential for virulence ${ }^{(11,12)}$, and $\operatorname{Ldt}_{\mathrm{Mt} 1}$ is believed to remodel the cell wall for survival in dormancy ${ }^{(8,13)}$.

LDTs and PBPs have different $\beta$-lactam susceptibilities, as evidenced by enzymatic, biochemical, and structural studies ${ }^{(14-20)}$. For example, PBPs are inactivated by covalent modification at a catalytic serine by $\beta$-lactam antibiotics (e.g., penams, monobactams, cephems, and carbapenems). In contrast, LDTs are inhibited by compounds in the carbapenem class, which bind to an active-site cysteine by thioester bond formation (Figure 1). Since the majority of cross-links in Mtb are formed by LDTs, this suggests that carbapenems could be effective drugs for treating $\operatorname{TB}^{(10,21,22)}$. The Mtb LDTs and PBPs are summarized in Table 1.
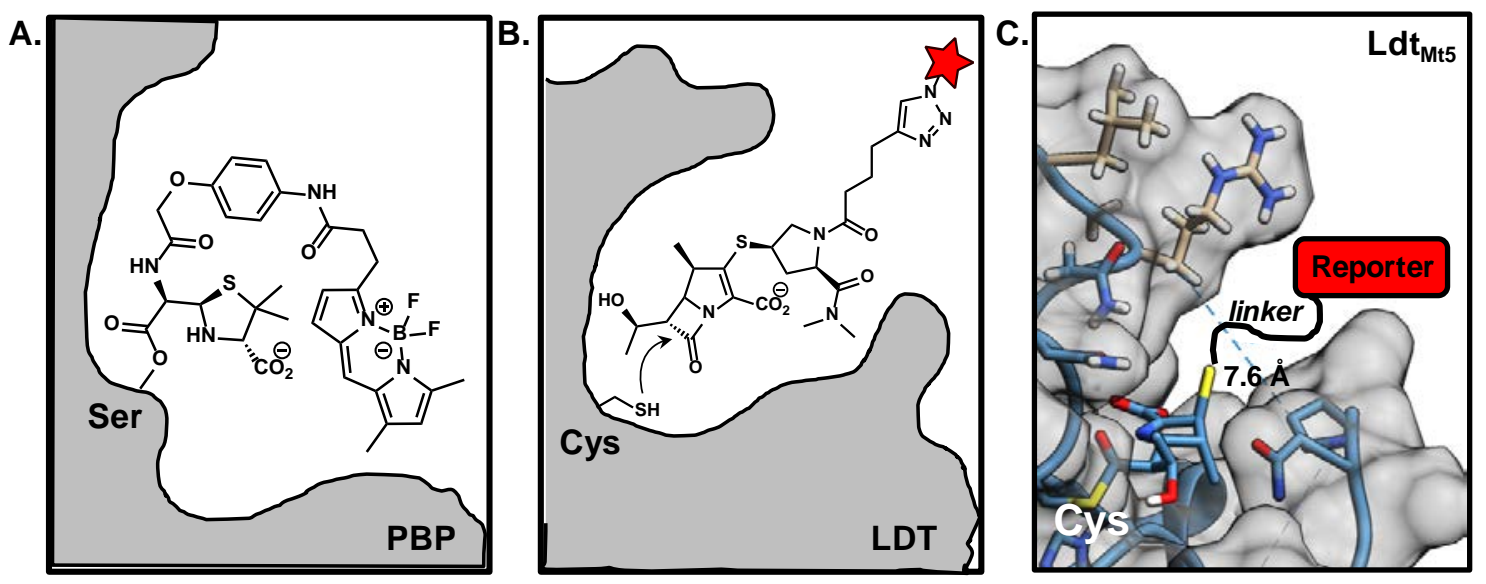

Figure 1. Active site labeling of PBPs and LDTs with $\beta$-lactam probes. A. In PBPs, the active-site serine binds to a reactive $\beta$-lactam probe, as shown for Bocillin FL. B. In contrast, the LDT's active-site cysteine binds to carbapenems (e.g., Mero-Cy5). C. Structure of the Ldt $_{M+5}$ active-site bound to a fragment of meropenem (PDB ID: 4ZFQ) ${ }^{(14)}$. The Mero-Cy5 probe, described herein, was designed to bind to LDTs with a reporter extending out of the active site, as illustrated.

$\beta$-lactams are not part of the standard treatment regimen for TB. However, the reason for this might lie in outdated observations made 70 years ago, shortly after the introduction of penicillin. A study from 1949 described a "penicillinase" that made $M t b$ resistant to $\beta$-lactam antibiotics ${ }^{(23)}$. This resistance was conferred by BlaC, a genomically-encoded $\beta$-lactamase ${ }^{(24,25)}$. The discovery of BlaC suggested that $\beta$ lactam antibiotics would be ineffective in treating TB. BlaC also inactivated the cephalosporins introduced in the $1960 \mathrm{~s}^{(26)}$. Yet, as reviewed by Story-Roller and Lamichhane ${ }^{(22)}$, the carbapenem class targets LDTs, which may provide distinct druggable targets in Mtb. BlaC has limited activity against carbapenems $^{(27-29)}$.

There is some evidence that $\beta$-lactams are therapeutically-relevant drugs for $\operatorname{TB}^{(22)}$. There have been occasional reports of successfully treating TB patients with co-administration of $\beta$-lactams with clavulanate, a BlaC inhibitor ${ }^{(21,30-36)}$. A 1998 report described using amoxicillin/clavulanate to cure multidrug resistant $\mathrm{TB}^{(35)}$. Drug-resistant $\mathrm{TB}$ has also been treated with imipenem ${ }^{(37)}$ and meropenem/clavulanate ${ }^{(30-32,34,36)}$. The latter treatment cured extensively-drug resistant TB patients $(83 \% \text { cure rate })^{(34)}$. This combination was also effective against dormant $M t b^{(38,39)}$. 
Table 1. $\beta$-lactam targets in Mtb.

\begin{tabular}{|c|c|c|c|}
\hline & Name & Rv \# & MW (kDa) \\
\hline \multirow{5}{*}{$\stackrel{n}{\circ}$} & $\mathrm{Ldt}_{\mathrm{Mt} 1}$ & Rv0116c & 26.9 \\
\hline & $\mathrm{Ldt}_{\mathrm{Mt} 2}$ & Rv2518c & 43.4 \\
\hline & $\mathrm{Ldt}_{\mathrm{Mt} 3}$ & Rv1433 & 28.7 \\
\hline & $\mathrm{Ldt}_{\mathrm{Mt} 4}$ & Rv0192 & 38.9 \\
\hline & $\mathrm{Ldt}_{\mathrm{Mt5}}$ & Rv0483 & 47.9 \\
\hline \multirow{11}{*}{$\begin{array}{l}n \\
0 \\
0 \\
0\end{array}$} & PonA1 & Rv0050 & 71.1 \\
\hline & PonA2 & Rv3682 & 84.6 \\
\hline & PBPA & Rv0016c & 51.6 \\
\hline & PBPB & Rv2163c & 72.5 \\
\hline & PBP-lipo & Rv2864 & 63.0 \\
\hline & PBP4 & Rv3627c & 46.8 \\
\hline & DacB1 & Rv3330 & 41.7 \\
\hline & DacB2 & Rv2911 & 29.7 \\
\hline & - & Rv0907 & 56.7 \\
\hline & - & Rv1367 & 41.3 \\
\hline & BlaC & Rv2068c & 32.6 \\
\hline
\end{tabular}

One outcome of the prior studies is a renewed interest in identifying $\beta$-lactams that can cure drugresistant TB. The scarcity of information on their protein targets in Mtb makes the development of broadly successful treatment regimens problematic. Furthermore, there is almost no information on which PBPs and LDTs are functional in dormant and actively replicating Mtb. Prior transcriptional analyses suggest that these enzymes are dynamically regulated by the pathogen based on its metabolic state ${ }^{(13,40)}$. However, transcript levels do not always correlate with enzyme activity.

Evaluating multiple enzymes at once is challenging using conventional biochemical or genetic approaches. Activity-based probes (ABPs) enable such studies. ABPs consist of a reporter linked to an irreversible inhibitor, which provides specificity for the enzyme target ${ }^{(41-43)}$. There is established precedence for using ABPs to assign peptidase functions in other bacteria ${ }^{(44-49)}$.

In this work, we used ABPs to profile the regulation of Mtb PBPs and LDTs. We produced a small set of structurally-diverse $\beta$-lactam probes, including a new meropenem derivative, to illuminate drugbound targets in protein gel-resolved mycobacterial lysates. We identified enzymes associated with 
both dormant and actively-replicating $M t b$. Our results suggest that carbapenems, including meropenem, could be useful drugs for treating latent or active TB infections.

\section{Results and Discussion}

\section{Identification of $\beta$-lactam targets encoded in the Mtb genome.}

We initiated this project by generating a comprehensive list of Mtb enzymes with active sites likely to interact with a $\beta$-lactam. We searched the Mtb H37Rv genome for genes with protein family (i.e., Pfam) annotation indicating potential PBP, LDT, or $\beta$-lactamase activity. ${ }^{(50)}$ This gave a total of 43 proteins with either validated or theoretical abilities to interact with $\beta$-lactams (see Supporting Information, Table S1). The abbreviated version of this list (Table 1) contains only the most likely $\beta$ lactam targets, and includes ten PBPs, five LDTs, and the $\beta$-lactamase $\operatorname{BlaC}^{(10)}$.

\section{Synthesis and validation of fluorescent $\beta$-lactam probes.}

Next, we assembled our chemical toolkit for detecting and identifying $\beta$-lactam targets in $M t b$ (Figure 2). For our studies, all ABPs contained a $\beta$-lactam antibiotic as the inhibitor. For a penam compound we obtained Bocillin-FL, a commercially-available green fluorescent penicillin ${ }^{(44)}$. We additionally selected monobactam, cephalosporin, and carbapenem scaffolds for structural diversity. An alkyne handle was included in these ABPs to enable ready access to fluorescent probes via azidealkyne conjugation.

A.

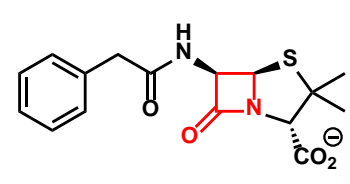

penicillin G

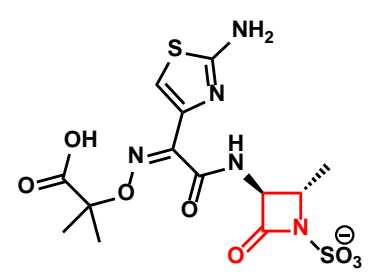

aztreonam

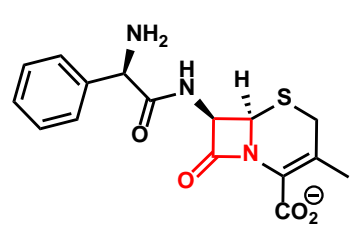

cephalexin

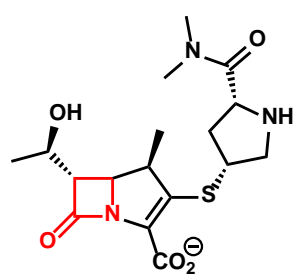

meropenem

B.

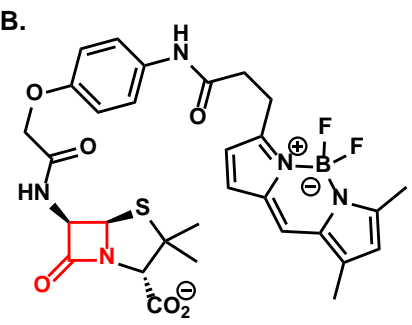

Bocillin-FL

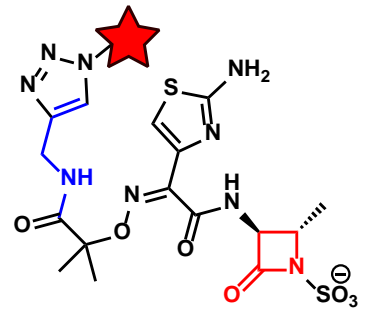

aztreonam-Cy5 (Azt-Cy5)
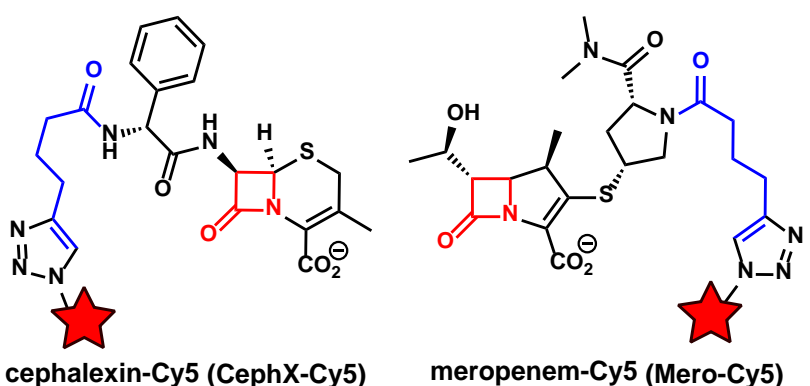

meropenem-Cy5 (Mero-Cy5)

Figure 2. Structures of $\beta$-lactam antibiotics (A) and activity-based probes (B) used in the current work. The Cy5conjugated derivatives were obtained by reacting the alkyne probe with sulfo-Cy5-azide to produce Azt-Cy5, CephX-Cy5, and Mero-Cy5. The red star signifies the sulfo-Cy5 fluorophore.

For the monobactam compound, we synthesized the known aztreonam-N-alkyne ${ }^{(48)}$. We found that increasing the reaction time resulted in a substantially higher yield of aztreonam-alkyne than previously reported (>95\% yield in $3 \mathrm{~d}$ vs. $19 \%$ in $30 \mathrm{~min}) .{ }^{48)}$ For the cephalosporin and carbapenem scaffolds we synthesized two new compounds: cephalexin-alkyne and meropenem-alkyne, respectively. For the 
cephalosporin probe, we used the readily available cephalexin as the starting material, providing a cost benefit over the more traditional cephalosporin $C .^{(46,48)}$ We synthesized cephalexin-alkyne under basic conditions similar to ones used by Carlson and co-workers to synthesize cephalosporin C probes. ${ }^{(46)} \mathrm{A}$ modification of these conditions to use a heterogeneous base $\left(\mathrm{Cs}_{2} \mathrm{CO}_{3}\right)$ generated meropenem-alkyne from meropenem. We selected meropenem as our carbapenem-class probe because of its clinical significance for treating TB. Also, it was straightforward to modify, unlike faropenem and imipenem. These alkynes readily underwent copper-catalyzed click reactions with Sulfo-Cyanine5 (Cy5) azide to generate far-red fluorescent probes: aztreonam-Cy5 (Azt-Cy5), cephalexin-Cy5 (CephX-Cy5), and meropenem-Cy5 (Mero-Cy5).

We used Bocillin-FL for our initial evaluation of activity in Mtb lysates (Figure 3). Prior work used this probe to detect PBPs_including PonA2-in Mycobacterium smegmatis (Msmeg), a rapidly growing lab strain of mycobacteria. ${ }^{(51,52)}$ We used Bocillin FL to analyze lysates prepared from mid-log phase cultures of $M t b\left(\mathrm{mc}^{2} 6020\right)$, a $\Delta l y s A \Delta$ panCD auxotroph of $M t b \mathrm{H}_{3} \mathrm{Rv}^{(53)}$. Labeled lysates were resolved by SDS-PAGE and imaged on a fluorescence scanner to detect green fluorescence from the target-bound probe (Figure 3). Labeled proteins were nearly all over $50 \mathrm{kDa}$, despite ten of the targets in Table 1 being of a lower molecular weight. Labeling was reduced by pre-treatment with penicillin $G$ and other $\beta$-lactam antibiotics (see Supplementary Information; Figure S1). This competition experiment demonstrated that labeling was specific for proteins that bind to $\beta$-lactams. The fluorescent band intensity corresponded with activity, not relative abundance (see Figure S1B). We observed three auto-fluorescent bands in the green channel. Additionally, there were other drawbacks to using BocillinFL: (1) Bocillin-FL labeled less than half of the potential targets; (2) it labeled only two proteins under 50 $\mathrm{kDa}$; (3) penicillins are poor inhibitors of LDTs.

We overcame those drawbacks by using our far-red fluorescent ABPs: Azt-Cy5, CephX-Cy5, and Mero-Cy5. Again, Mtb lysates were probe-treated, resolved by SDS-PAGE, and imaged to detect Cy5labeled targets (Figure 3). Compared with Bocillin-FL, each of these probes showed more fluorescent bands. They also labeled multiple proteins with an apparent molecular weight of less than $50 \mathrm{kDa}$. We performed competition experiments with the parent antibiotic. Labeling was reduced under these conditions, indicating that the labeling was specific for proteins that bind $\beta$-lactams. Notably, these new probes each revealed distinct target selectivity plus bands that were undetected by Bocillin-FL. All four probes detected BlaC activity (see next section).

\section{Identification of protein targets of Mero-Cy5 from gel-excised bands.}

We initiated the process of identifying proteins that were labeled by Mero-Cy5 because this compound provided the clearest in-gel activity patterns. In these studies we compared lysates from actively-replicating cultures with dormant cultures of Mtb. We induced dormancy by incubation under hypoxic conditions, as described ${ }^{(54-56)}$. Mtb lysates were fractionated by centrifugation (into pellet and supernatant ${ }^{(57)}$ ), labeled with Mero-Cy5, and resolved by SDS-PAGE. Fluorescent bands were excised and submitted for identification by liquid chromatography tandem mass spectrometry (LC-MS/MS). Peptides were considered positively identified if their probability of identification was $\geq 99 \%$ with a falsediscovery rate (FDR) of $0.67 \%$. Proteins were considered positively identified if two or more exclusive unique peptides were detected (probability of identification $\geq 95 \%$; FDR $0.0 \%$ ). 


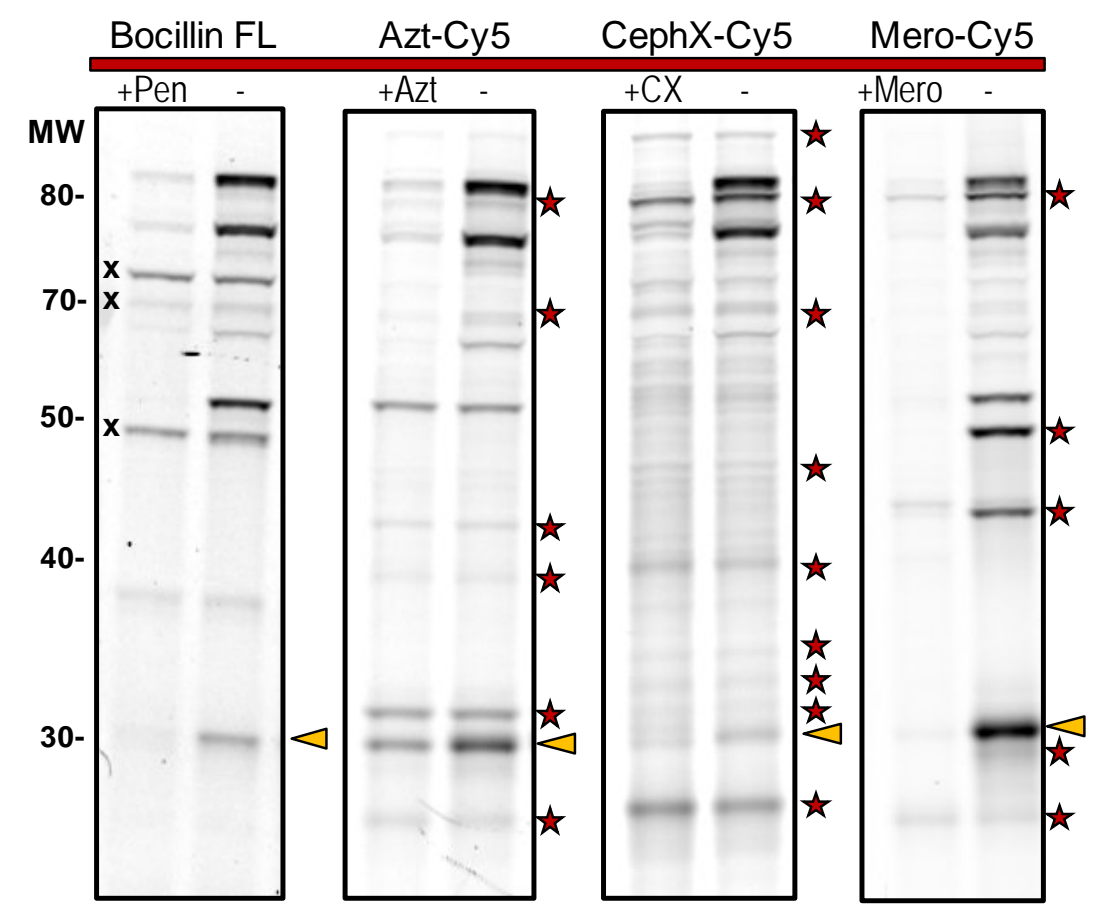

Figure 3. $\boldsymbol{\beta}$-lactam probes covalently bind to $\boldsymbol{M t b}$ protein targets, enabling fluorescent detection. Lysates were treated with probe, resolved by SDS-PAGE, and imaged. Three auto-fluorescent bands $(x)$ were observed with Bocillin FL, but not with Cy5 probes. Labeling was reduced by pre-treatment with antibiotic (left lanes). Pen: Penicillin G; Azt: Aztreonam; CX: Cephalexin; Mero: Meropenem. $\star$ : Targets revealed by Azt-Cy5, CephX-Cy5,or Mero-Cy5 (but not Bocillin FL). BlaC activity: 4 .

We narrowed our analysis to proteins with putative PBP, LDT, or $\beta$-lactamase activity (see Table S1). Of the forty-three proteins in Table S1, thirty-two of them were identified in our samples (Table S2). Specifically, we identified transpeptidases (PonA1, PonA2, PBPA, LDT $\mathrm{Mt}_{2}, \mathrm{LDT}_{\mathrm{Mt} 3}, \mathrm{LDT}_{\mathrm{Mt} 5}$ ), carboxypeptidases (DacB1, DacB2, PBP4), and the $\beta$-lactamase BlaC. The remaining proteins detected were confirmed or putative $\beta$-lactamases based on Pfam annotation. We attempted to precisely extract bands based on fluorescent signal, but the presence of a particular protein does not indicate that it was a source of the Cy5 signal in a given region. Experimental details and the full data set are provided in the SI, including annotated gels from which we excised bands (Figure S2).

Band identifications are provided in Figures 4-6. For clarity, bands were divided into three regions based on migration and molecular weight: high ( $80 \mathrm{kDa})$, middle (40-50 kDa), and low (15-30 kDa), as indicated in Figure 4A. First we analyzed bands that migrated at or below $30 \mathrm{kDa}$. We determined that BlaC activity most likely contributes much of the signal at $30 \mathrm{kDa}$ in normoxic samples (Band ID: 10NP8). We identified 18 unique peptides from BlaC with $69.7 \%$ sequence coverage. We validated our assignment by comparing lysates from Mtb $\mathrm{mc}^{2} 6020$ with Mycobacterium marinum, a close genetic relative, and with Msmeg ( $\left.\mathrm{mc}^{2} 155\right)$, a species that lacks a BlaC ortholog (Figure S3). The major $\beta$ lactamase in Msmeg, BlaS (31 kDa), is not a target of this inhibitor ${ }^{(24,58,59)}$. We observed a clavulanatesensitive band at $30 \mathrm{kDa}$ for lysates from Mtb and M. marinum, but not Msmeg. This comparative analysis provides compelling evidence in support of this assignment. 


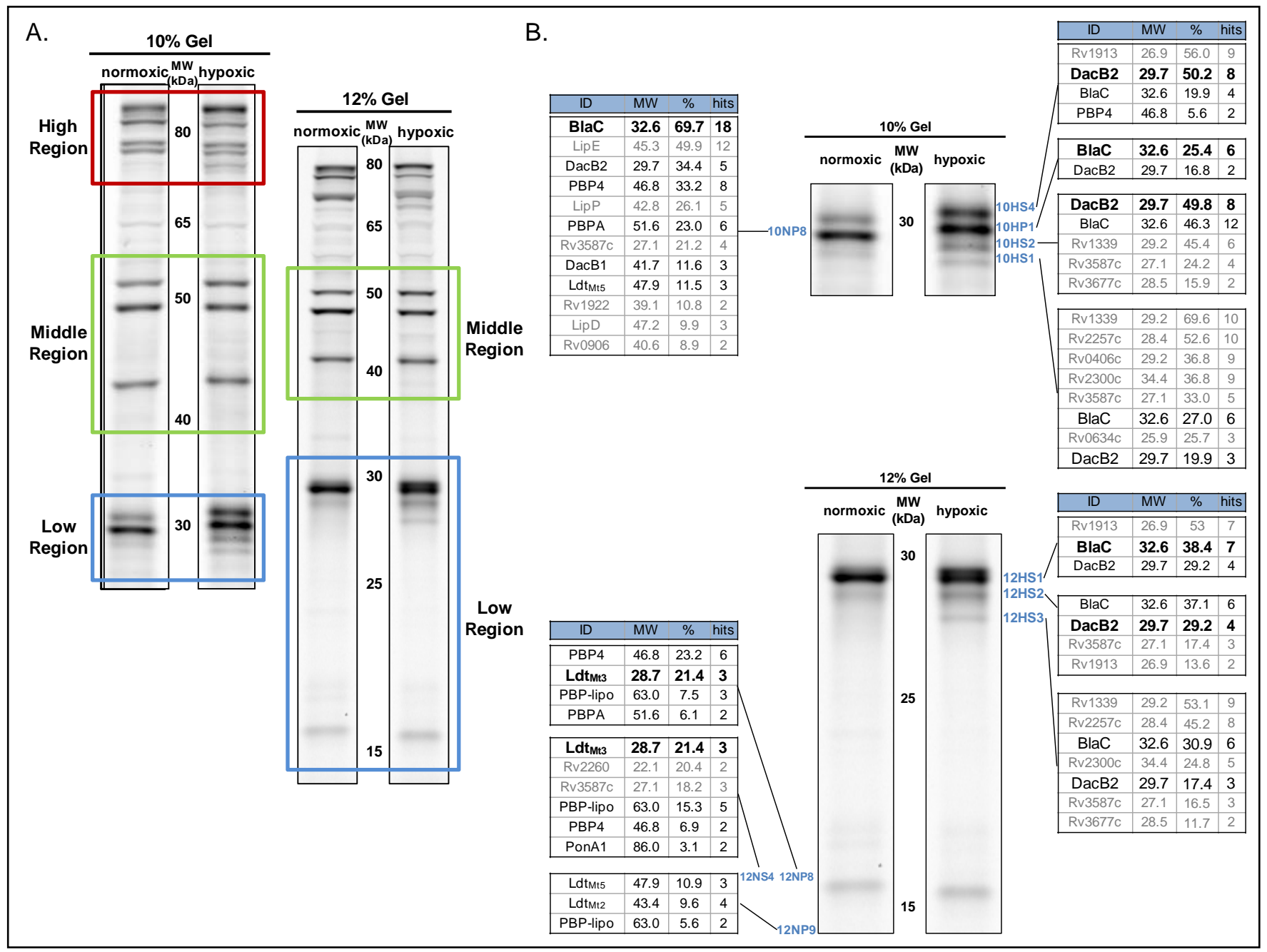

Figure 4. Analysis of lysates for MS-based identification of proteins labeled with Mero-Cy5. A. Visualization of the division of protein gels into High, Middle, and Low regions. B. Protein identifications for bands excised from the "low region" of the $10 \%$ (top) and 12\% (bottom) SDS-PAGE gels. The most likely source of the fluorescent signal observed is emphasized in Bold. Known targets of $\beta$-lactams are indicated in black, while putative targets are in gray.

Interestingly, we observed a different banding pattern near $30 \mathrm{kDa}$ for proteins obtained from normoxic versus hypoxic cultures (Figure 4B). We excised three of the $12 \%$ gel bands from hypoxic samples (10HS1, 10HS2, and 10HS4); all three contained BlaC peptides. Peptides from the carboxypeptidase DacB2 and other $\beta$-lactamases were also identified in these excised bands. Similar results were obtained from four bands excised from the $10 \%$ gel. This made the source of the signal ambiguous because it could be from any combination of BlaC, DacB2, or the putative $\beta$-lactamases. While it has been shown that BlaC represents the majority of $\beta$-lactamase activity under regular growth conditions $^{38}$, similar studies have not been conducted with non-replicating bacteria. The presence of non-BlaC $\beta$-lactamases in dormant bacteria could alter their resistance profile toward various classes of $\beta$-lactam antibiotics.

The bands representing the lowest molecular weight proteins were excised from a $12 \%$ gel. Based on size, the signal near $15 \mathrm{kDa}$ (band ID 12NP8) is most likely derived from Mero-Cy5 bound to $\mathrm{Ldt}_{\mathrm{Mt} 3}$ (28.7 kDa). Ldt $\mathrm{Mt}_{3}$ was also found in a band excised from another normoxic sample (12NS4). We 
sometimes observed other fluorescent bands in the 15-20 kDa region of the gel (see Figure 6), but those proteins were less stable to storage and electrophoresis and we were unable to identify them.

Next we analyzed the middle region of the gel, which contained three fluorescent bands between 40 and $50 \mathrm{kDa}$ (Figure 5). The two bright bands near $50 \mathrm{kDa}$ were observed in both hypoxic and normoxic samples. These bands provided peptide hits from nine proteins. Based on molecular weight, we deduced that these two bands most likely contain PBPA (51.6 kDa), PBP4 (46.8 kDa), or LDT Mt5 $_{\text {(47.9 }}$ $\mathrm{kDa}$ ). We believe that the upper band is PBPA based on \% coverage and the number of peptide hits. When we compared the gel images from different $\beta$-lactam probes (see Figure 3 ), the band just below $50 \mathrm{kDa}$ was only prominent with Mero-Cy5. This implies that the target can hydrolyze and release monobactam, cephalosporin, and/or penicillin based compounds-but not carbapenems ${ }^{27,48}$. We therefore believe that this signal was generated by $\mathrm{Ldt}_{\mathrm{Mt5}}$.

Analysis of the band just above $40 \mathrm{kDa}$ resulted in fourteen proteins with relevant Pfam notation. For these, eight were putative $\beta$-lactamases, four were PBPs (PBP4, DacB1, PBPA, and PonA2) and two were LDTs (Ldt $\mathrm{Mt}_{\mathrm{M} 5}$ and $\left.\mathrm{Ldt}_{\mathrm{Mt} 2}\right)$. By molecular weight, DacB1 (41.7 kDa) and $\mathrm{Ldt}_{\mathrm{Mt} 2}(43.4 \mathrm{kDa})$ are the most likely to be present in this region. $\mathrm{Ldt}_{\mathrm{Mt} 2}$ was identified from three bands $(10 \mathrm{NP} 7,10 \mathrm{HP} 2$, and 12NP3), corroborating its presence near $40 \mathrm{kDa}$.

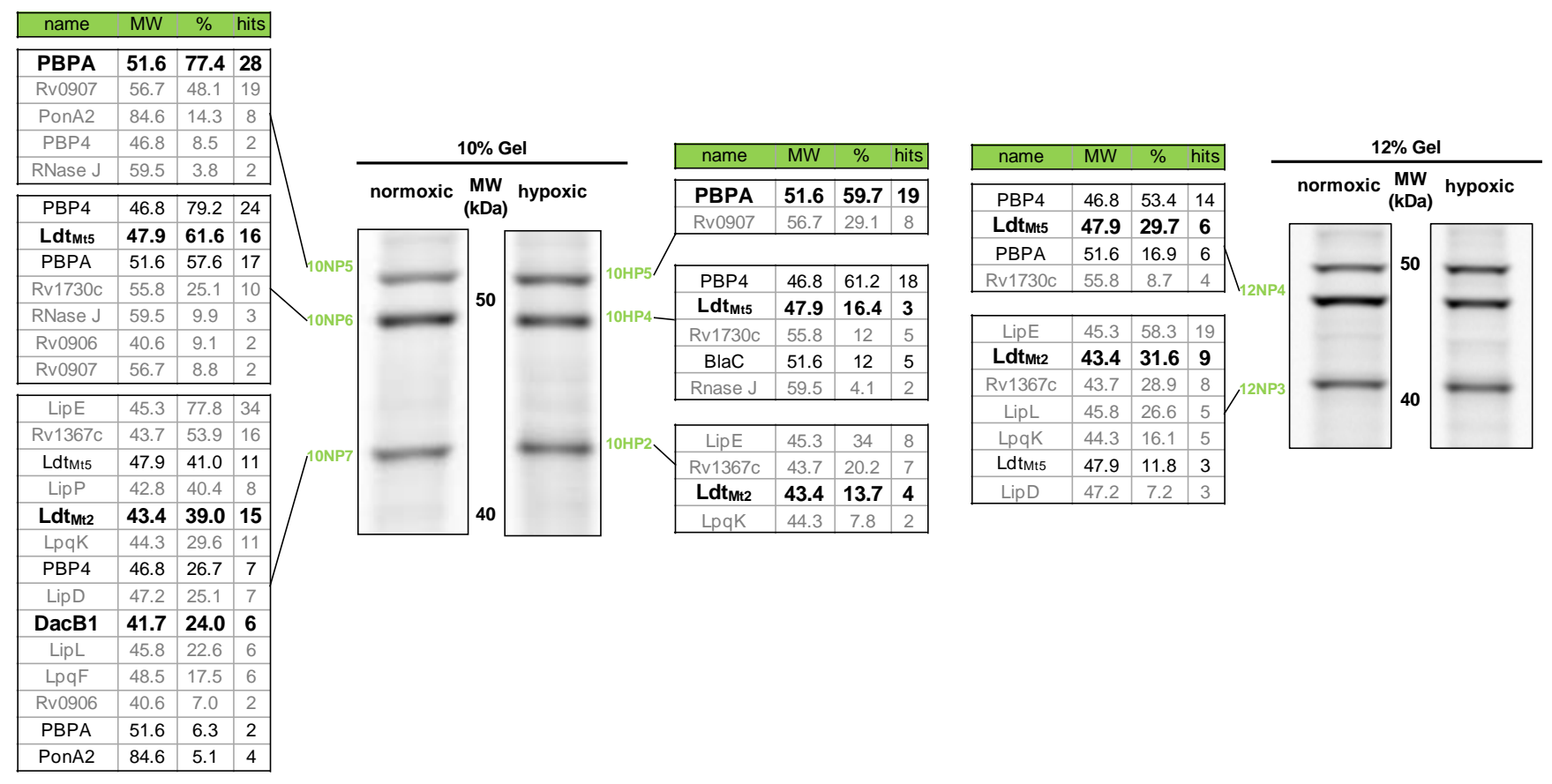

Figure 5. Identification of proteins associated with fluorescent bands observed between $\mathbf{4 0}$ and $\mathbf{5 0}$ kDa. Protein identifications for bands excised from the "middle region" of the $10 \%$ (left) and 12\% (right) SDS PAGE gels. The most likely source of the fluorescent signal observed is provided in Bold. Known targets of $\beta$-lactams are indicated in black, while putative targets are in gray. 


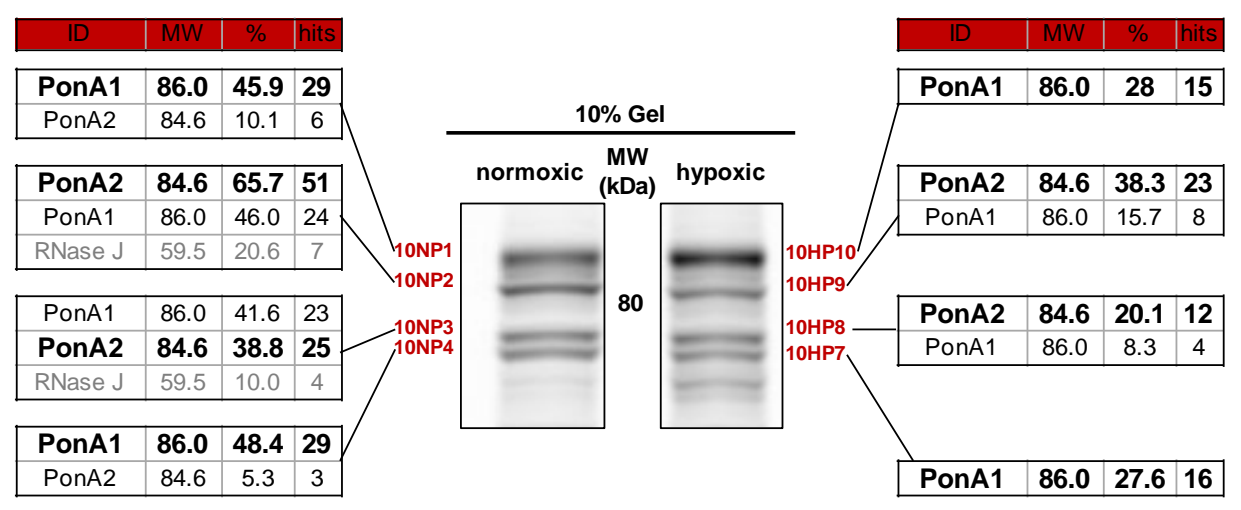

Figure 6. Identification of proteins associated with fluorescent bands near $\mathbf{8 0} \mathbf{~ k D a}$. Protein identifications for bands excised from a 10\% SDS PAGE gel. Two PBPs, PonA1 and PonA2, were identified by LC-MS/MS. The most likely source of the fluorescent signal observed is provided in Bold.

From the "high region", we analyzed bands from near $80 \mathrm{kDa}$ (Figure 6). We obtained peptide hits from normoxic and hypoxic samples, with good sequence coverage for both PonA1 and PonA2. We believe that the signal in the top and bottom of the four bands is from PonA1. The appearance of PonA1 in two locations is unsurprising because it is regulated by phosphorylation, which can influence protein migration by SDS-PAGE ${ }^{(52,60)}$. In hypoxia, the top band (10HP10) is more fluorescent than the bottom one (10HP7), which may indicate that PonA1 is differentially modified in dormancy. We believe that the two middle bands (10NP2 and 10NP3) contain predominantly PonA2, with the lower band resulting from cleavage of a $3 \mathrm{kDa}$ signal sequence ${ }^{(61)}$. The signal peptide was only identified in the upper band (10NP2).

To summarize, we used LC-MS/MS to identify five PBPs (PonA1, PonA2, PBPA, DacB1, and DacB2), BlaC, and three LDTs (LDT $\left.\mathrm{Lt}_{\mathrm{Mt}}, \mathrm{LDT}_{\mathrm{Mt} 3}, \mathrm{LDT}_{\mathrm{Mt}}\right)$. We also found evidence of many putative $\beta$ lactamases. Future studies might use purified proteins or genetic knock-out strains to more definitively assign activities identified here. Alternatively, samples could be treated with a biotinylated ABP and affinity-enriched before MS-based analysis. That approach is useful for enriching rare targets before LC-MS/MS analysis. Overall, our results demonstrate the utility of mero-Cy5 for identifying $\beta$-lactam drug targets in both normoxic and hypoxic Mtb.

\section{Patterns of protein activity from hypoxic and normoxic Mtb.}

Our MS analysis suggested that the activity of PBPs and LDTs changes in response to hypoxia. This fits prior observations of that Mtb increases the LDT-mediated cross-links in dormancy ${ }^{(11,62,63)}$. We investigated enzyme regulation further using ABP-labeled lysates from hypoxic and normoxic (actively-replicating). In Figure 7, the most plausible protein identifications are annotated based on our MS analysis.

Mero-Cy5 revealed changes in PBP and LDT activity in dormancy. We found that many targets were present-if at lower intensity-in both growth conditions (stars; $\star$ ). For example, PonA1, PonA2, $\mathrm{LDT}_{\mathrm{Mt} 3}$, and $\mathrm{LDT}_{\mathrm{Mt5}}$ maintained their activity levels in dormancy. Others targets, particularly between 40 and $80 \mathrm{kDa}$, were less active in dormancy and at least three bands were absent (black dash-mark; -). The cephalexin probe indicated some up-regulation of faint bands in the $80 \mathrm{kDa}$ region of the gel (Figure S4). These changes were also visible with Azt-Cy5, Mero-Cy5, and Bocillin-FL (Figure S4). It 
would be interesting to know if these changes are a result of post-translational modifications, such as phosphorylation ${ }^{(52)}$. Bocillin-FL probe offered little insight for targets below $50 \mathrm{kDa}$, as expected.

As noted in the previous section, we observed up-regulated enzyme activity near $30 \mathrm{kDa}$ from hypoxic lysates. In particular, the $\sim 28 \mathrm{kDa}$ band was only distinct in hypoxic samples. This region was also notable in lysates treated with Azt-Cy5, which showed similar up-regulation of bands from 25-30 $\mathrm{kDa}$ under hypoxia (Figure S4). Our proteomics results indicate that DacB2 migrates in this region of the gel. This suggests that DacB2 could have enhanced activity under hypoxic conditions, an interesting result consistent with a prior proteomics study ${ }^{(40)}$. To summarize, our in-gel analysis provides evidence that the PBPs and LDTs are dynamically regulated in response to hypoxia.

\section{Drug susceptibility of PBPs and LDTs in Mtb}

We set out to investigate antibiotic susceptibility in Mtb using ABPs. For these studies, we analyzed ABP-labeled lysates alongside lysates pre-treated with a selection of $\beta$-lactam antibiotics as inhibitors (Figure 6). Again, we studied lysates from dormant (hypoxic) and actively-replicating cultures. After drug-treatment, lysates were labeled with Mero-Cy5. As an additional control we exposed lysates to a sulfo-Cy5 conjugated to 5-hexynoic acid instead of a $\beta$-lactam (Lane 12). The absence of labeling with this compound indicated that the $\beta$-lactam, not Cy5, dictated target binding specificity. We tested cephalexin, ceftriaxone, aztreonam, penicillin G, ampicillin, carbenicillin, meropenem, faropenem, and clavulanate. All of these drugs except faropenem are prescribed for treating bacterial infections in the United States.
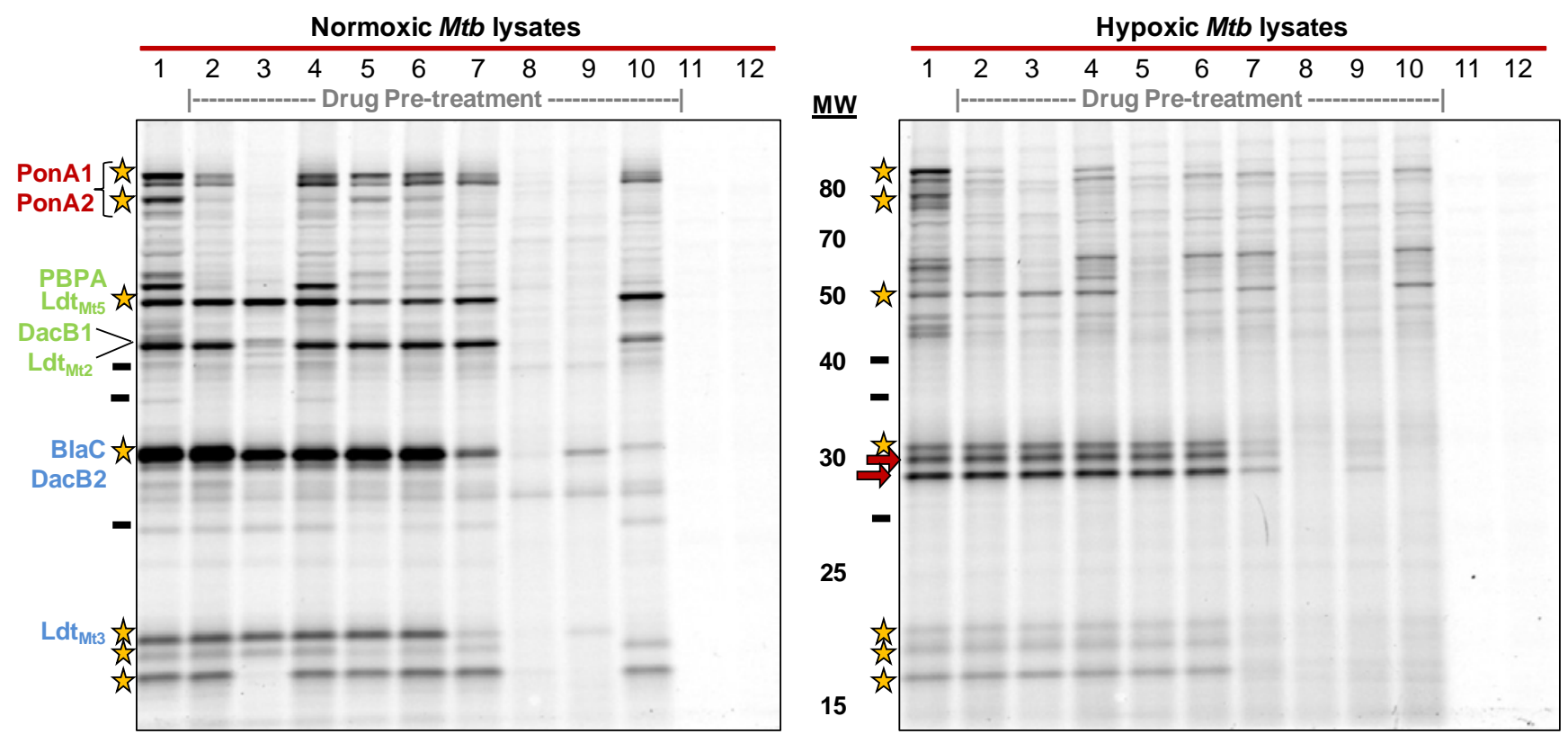

Figure 7. Mero-Cy5 reveals enzyme regulation and drug targets in lysates obtained from normoxic and hypoxic Mtb. Lane 1: No drug pre-treatment. Lanes 2-10: Drug pre-treatment. 2: Cephalexin; 3: Ceftriaxone; 4: Aztreonam; 5: Penicillin G; 6: Ampicillin; 7: Carbenicillin; 8: Meropenem; 9: Faropenem; 10: Clavulanate. Lane 11: Lysates not treated with Mero-Cy5. Lane 12: Lysates treated with a Cy5-(triazole)-butanoic acid. Putative protein identities were obtained by MS analysis. Legend: Gold stars denote enzymes that retain activity under hypoxic conditions. Red arrows indicate bands with enhanced activity in hypoxia. Black dash-marks indicate bands that are absent in hypoxia, but present under normoxia. 
We were most interested in identifying bands that are targets of the carbapenems (i.e., meropenem and faropenem) because these drugs inhibit LDTs ${ }^{(22)}$. As expected, pre-treatment with meropenem (Lane 8), the closest structural analog to Mero-Cy5, eliminated most of the ABP's labeling. We observed that meropenem completely inhibited a band that corresponds to $\mathrm{Ldt}_{\mathrm{Mt} 5}$, which is consistent with Lamichhane et al.'s finding that meropenem acylates the $\mathrm{Ldt}_{\mathrm{Mt} 5}$ active site ${ }^{(14)}$. Similar results were found with faropenem (Lane 9).

Ceftriaxone (Lane 3), a clinically-approved cephem, was more effective than cephalexin (Lane 2) at blocking Mero-Cy5 binding, although neither antibiotic was an effective inhibitor of BlaC, $\mathrm{Ldt}_{\mathrm{Mt5}}$, or $\mathrm{Ldt}_{\mathrm{Mt3}}$. The penams, penicillin $\mathrm{G}$ (Lane 5), ampicillin (Lane 6), and carbenicillin (Lane 7), were less effective at inhibiting targets than other drugs, but reduced Mero-Cy5 labeling of PBPA. BlaC, which retained some activity in dormancy, was inhibited by several drugs (Lanes 7-10), including clavulanate (Lane 10) and meropenem (Lane 8).

Antibiotic susceptibility was also analyzed using Azt-Cy5 and CephX-Cy5, and we observed similar inhibition profiles (Figure S5). Overall, our findings indicate that PBP and LDT activities can be reduced or eliminated in vitro using clinically-approved $\beta$-lactams.

\section{Conclusion}

In conclusion, we used fluorescent $\beta$-lactam probes to identify active PBPs, LDTs, and $\beta$ lactamases in Mtb. In a direct comparison with Bocillin-FL, we found that Azt-Cy5, CephX-Cy5, and Mero-Cy5 enabled more targets to be detected in PAGE-resolved lysates. Although we collected data with Cy5-modified probes, we reported the synthesis of the alkyne derivatives because they enable facile modification with an assortment of reporters. For example, a biotinylated probe would enable enrichment of $\beta$-lactam targets before proteomic analysis ${ }^{(55)}$.

We used Mero-Cy5, a new probe, to identify 32 proteins, including transpeptidases (PonA1, PonA2,

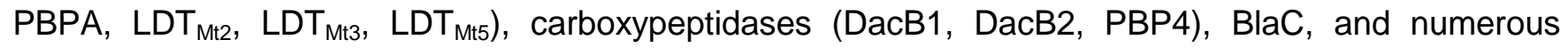
putative $\beta$-lactamases (see Table S2). We did not identify $\mathrm{LDT}_{\mathrm{Mt} 1}$ or $\mathrm{LDT}_{\mathrm{Mt} 4}$, although there is structural evidence that both LDTs bind carbapenems ${ }^{(15-17)}$. We found evidence that post-translational modifications alter the migration patterns observed in-gel. In future work, it would be interesting to study in more detail how various post-translational modifications alter PBP $^{(52)}$ and LDT activities during different stages of infection.

We used Mero-Cy5 to investigate enzyme regulation in dormant and actively-replicating samples. We found numerous changes in fluorescent banding patterns and intensity between the two states. For example, we found that some bands (25-30 kDa) were up-regulated under hypoxia. A priority for future work is to identify the exact source of that activity because it is unclear if those bands are attributable to BlaC. Lastly, we treated lysates with a set of $\beta$-lactam drugs in clinical use today and found that meropenem and faropenem were both good inhibitors of BlaC, PBPs, and LDTs. Overall, we demonstrated that mero-Cy5 is a useful probe for target identification, analysis of enzyme regulation, and determining enzyme inhibition by various $\beta$-lactam drugs. 


\section{Methods}

\section{Synthesis of activity-based probes}

Bocillin FL was obtained from ThermoFisher Sci. Azt-alkyne, CephX-alkyne, and Mero-alkyne were synthesized from the parent $\beta$-lactam, as described in the Supporting Information. The fluorescent probes were generated in vitro via Cu-catalyzed click reaction (1h, room temperature) of the alkyne $(100 \mu \mathrm{M})$ with sulfo-Cyanine5-azide $(100 \mu \mathrm{M}), 1.25 \mathrm{mM} \mathrm{THTPA} 0.25 \mathrm{mM} \mathrm{CuSO}_{4}$, and sodium ascorbate (15 mM) in HEPES buffer (pH 7.3).

\section{Mtb culture conditions}

Mtb $\mathrm{mc}^{2} 6020$ was grown in Middlebrook 7H9-OADC medium supplemented with lysine, pantothenate, and casamino acids. For normoxic cultures the flasks were maintained at $37{ }^{\circ} \mathrm{C}$ with $100 \mathrm{rpm}$ shaking under atmospheric conditions. For hypoxic cultures, mid-log phase cultures were diluted to $\mathrm{OD}_{600} 0.4$ with additional medium and grown as standing cultures under $1 \% \mathrm{O}_{2}$ and $5 \% \mathrm{CO}_{2}$ at $37^{\circ} \mathrm{C}$.

\section{Preparation of lysates}

Mtb grown under normoxic conditions was harvested at mid-log phase $\left(\mathrm{OD}_{600}=0.9-1.2\right)$. Mtb grown under hypoxic conditions was harvested at fixed time-points $\left(\mathrm{OD}_{600}=0.4-0.8\right)$. All cells were collected by centrifugation (10 $\mathrm{min}, 4,000 \times \mathrm{g}, 4^{\circ} \mathrm{C}$ ) and washed twice with PBS containing $0.05 \%$ Tween 80 . Pellets were resuspended in detergent-free lysis buffer [50 mM Tris (pH 7.5 at $\left.4{ }^{\circ} \mathrm{C}\right), 50 \mathrm{mM} \mathrm{NaCl}, 0.5$ $\mathrm{mM} \mathrm{CaCl}_{2}, 0.5 \mathrm{mM} \mathrm{MgCl}_{2}$ ], lysed by mechanical disruption, and pelleted by centrifugation (10 min, $\left.4,000 \times \mathrm{g}, 4^{\circ} \mathrm{C}\right)$. The supernatant was transferred to a separate tube and the beads and cell debris resuspended in an equivalent volume of detergent containing lysis buffer [50 mM Tris ( $\mathrm{pH} 7.5$ at $\left.4{ }^{\circ} \mathrm{C}\right)$, $50 \mathrm{mM} \mathrm{NaCl}, 0.5 \mathrm{mM} \mathrm{CaCl}_{2}, 0.5 \mathrm{mM} \mathrm{MgCl}_{2}, 0.4 \%$ triton X-100] and the mechanical disruption and centrifugation steps were repeated. The combined supernatants were pelleted by centrifugation (10 $\min , 4,000 \times \mathrm{g}, 4^{\circ} \mathrm{C}$ ) to remove insoluble debris and sterilized by filtration ( $0.2 \mu \mathrm{m}$ PES membrane).

\section{Lysate labeling and imaging}

Lysates were diluted with detergent containing lysis buffer [50 mM Tris (pH 7.5 at $4{ }^{\circ} \mathrm{C}$ ), $50 \mathrm{mM} \mathrm{NaCl}$, $0.5 \mathrm{mM} \mathrm{CaCl}_{2}, 0.5 \mathrm{mM} \mathrm{MgCl}_{2}, 0.2 \%$ triton X-100] to normalize the total protein concentration. For samples pre-treated with antibiotics, the antibiotic was added for 15 min prior to probe-labeling. Lysates were labeled with the Cy5-modified probe $(5 \mu \mathrm{M})$ for $1 \mathrm{~h}$ at rt. Lysates were resolved by SDS-PAGE, washed, fixed, and imaged on a Typhoon multi-mode imager.

\section{Band excision and LC-MS/MS proteomics}

All bands were excised from an SDS-PAGE gel based on the fluorescent image and stored frozen. Gel slices were processed to remove SDS, reduced, and methylated. Proteins were trypsin-digested and the resulting peptides were concentrated. LC-MS/MS based proteomics was performed by the OHSU Proteomics Shared Resource Facility.

\section{Supporting information}

The Supporting Information includes detailed methods and Figures S1-S7. An Excel file containing Tables S1 and S2 are available by request (beattyk@ohsu.edu). 


\section{Acknowledgments}

Funding for this research was provided by the Knight Cancer Institute and the OHSU School of Medicine. S.R.L. was supported by an NIH T32 training grant (T32-AI07472). We are grateful to Dr. Gyanu Lamichhane (JHU) and Dr. Clifton Barry (NIH) for helpful discussions, Scotland Farley (OHSU) for synthesizing the aztreonam probe, and Dr. Kyle Gee (ThermoFisher Sci) for providing Bocillin FL.

\section{Citations}

1. World Health Organization (2018) Global Tuberculosis Report 2018, (Galbraith-Emami, s., Ed.) 20th ed., Geneva.

2. Barry, C. E., and Blanchard, J. S. (2010) The chemical biology of new drugs in the development for tuberculosis, Curr. Opin. Chem. Biol. 14, 456-466.

3. Wong, E. B., Cohen, K. A., and Bishai, W. R. (2013) Rising to the challenge: new therapies for tuberculosis, Trends Microbiol. 21, 493-501.

4. Zumla, A. I., Gillespie, S. H., Hoelscher, M., Philips, P. P., Cole, S. T., Abubakar, I., McHugh, T. D., Schito, M., Maeurer, M., and Nunn, A. J. (2014) New antituberculosis drugs, regimens, and adjunct therapies: needs, advances, and future prospects, The Lancet. Infectious diseases 14, 327-340.

5. Barry, C. E., Boshoff, H. I., Dartois, V., Dick, T., Ehrt, S., Flynn, J., Schnappinger, D., Wilkinson, R. J., and Young, D. (2009) The spectrum of latent tuberculosis: rethinking the biology and intervention strategies, Nat. Rev. Micro. 7, 845-855.

6. Bentrup, K. H. z., and Russell, D. G. (2001) Mycobacterial persistence: adaptation to a changing environment, Trends Microbiol. 9, 597-605.

7. Esmail, H., Barry, C. E., and Wilkinson, R. J. (2012) Understanding latent tuberculosis: the key to improved diagnostic and novel treatment strategies, Drug Discov. Today 17, 514-521.

8. Lavollay, M., Arthur, M., Fourgeaud, M., Dubost, L., Marie, A., Veziris, N., Blanot, D., Gutmann, L., and Mainardi, J.-L. (2008) The Peptidoglycan of Stationary-Phase Mycobacterium tuberculosis Predominantly Contains Cross-Links Generated by I,d-Transpeptidation, J. Bacteriol. 190, 4360-4366.

9. Wietzerbin, J., Das, B. C., Petit, J. F., Lederer, E., Leyh-Bouille, M., and Ghuysen, J. M. (1974) Occurrence of D-alanyl-(D)-meso-diaminopimelic acid and meso-diaminopimelyl-meso-diaminopimelic acid interpeptide linkages in the peptidoglycan of Mycobacteria, Biochemistry 13, 3471-3476.

10. Tolufashe, G. F., Sabe, V. T., Ibeji, C. U., Ntombela, T., Govender, T., Maguire, G. E. M., Kruger, H. G., Lamichhane, G., and Honarparvar, B. (2018) Structure and function of L,D- and D,D-transpeptidase family enzymes from Mycobacterium tuberculosis, Curr Med Chem.

11. Gupta, R., Lavollay, M., Mainardi, J.-L., Arthur, M., Bishai, W. R., and Lamichhane, G. (2010) The Mycobacterium tuberculosis protein LdtMt2 is a nonclassical transpeptidase required for virulence and resistance to amoxicillin, Nat Med 16, 466-469.

12. Schoonmaker, M. K., Bishai, W. R., and Lamichhane, G. (2014) Nonclassical transpeptidases of Mycobacterium tuberculosis alter cell size, morphology, the cytosolic matrix, protein localization, virulence, and resistance to beta-lactams, J Bacteriol 196, 1394-1402.

13. Betts, J. C., Lukey, P. T., Robb, L. C., McAdam, R. A., and Duncan, K. (2002) Evaluation of a nutrient starvation model of Mycobacterium tuberculosis persistence by gene and protein expression profiling, Mol. Microbiol. 43, 717-731.

14. Brammer Basta, L. A., Ghosh, A., Pan, Y., Jakoncic, J., Lloyd, E. P., Townsend, C. A., Lamichhane, G., and Bianchet, M. A. (2015) Loss of a Functionally and Structurally Distinct Id-Transpeptidase, LdtMt5, Compromises Cell Wall Integrity in Mycobacterium tuberculosis, J. Biol. Chem. 290, 25670-25685.

15. Cordillot, M., Dubée, V., Triboulet, S., Dubost, L., Marie, A., Hugonnet, J.-E., Arthur, M., and Mainardi, J.L. (2013) In Vitro Cross-Linking of Mycobacterium tuberculosis Peptidoglycan by I,d-Transpeptidases and Inactivation of These Enzymes by Carbapenems, Antimicrob. Agents Chemother. 57, 5940-5945.

16. Correale, S., Ruggiero, A., Capparelli, R., Pedone, E., and Berisio, R. (2013) Structures of free and inhibited forms of the I,d-transpeptidase LdtMt1 from Mycobacterium tuberculosis, Acta Crystallogr. D. 69, 1697-1706.

17. Dubée, V., Triboulet, S., Mainardi, J.-L., Ethève-Quelquejeu, M., Gutmann, L., Marie, A., Dubost, L., Hugonnet, J.-E., and Arthur, M. (2012) Inactivation of Mycobacterium tuberculosis I,d-Transpeptidase LdtMt1 by Carbapenems and Cephalosporins, Antimicrob. Agents Chemother. 56, 4189-4195. 
18. Erdemli, Sabri B., Gupta, R., Bishai, William R., Lamichhane, G., Amzel, L. M., and Bianchet, Mario A. (2012) Targeting the Cell Wall of Mycobacterium tuberculosis: Structure and Mechanism of L,DTranspeptidase 2, Structure 20, 2103-2115.

19. Filippova, E. V., Kieser, K. J., Luan, C. H., Wawrzak, Z., Kiryukhina, O., Rubin, E. J., and Anderson, W. F. (2016) Crystal structures of the transpeptidase domain of the Mycobacterium tuberculosis penicillinbinding protein PonA1 reveal potential mechanisms of antibiotic resistance, The FEBS journal 283, 22062218.

20. Kumar, P., Kaushik, A., Lloyd, E. P., Li, S. G., Mattoo, R., Ammerman, N. C., Bell, D. T., Perryman, A. L., Zandi, T. A., Ekins, S., Ginell, S. L., Townsend, C. A., Freundlich, J. S., and Lamichhane, G. (2017) Nonclassical transpeptidases yield insight into new antibacterials, Nat Chem Biol 13, 54-61.

21. Jaganath, D., Lamichhane, G., and Shah, M. (2016) Carbapenems against Mycobacterium tuberculosis: a review of the evidence, Int. J. Tuberc. Lung Dis. 20, 1436-1447.

22. Story-Roller, E., and Lamichhane, G. (2018) Have we realized the full potential of beta-lactams for treating drug-resistant TB?, IUBMB life 70, 881-888.

23. Iland, C. N., and Baines, S. (1949) The effect of penicillin on the tubercle bacillus: Tubercle penicillinase, J. Pathol. Bacteriol. 61, 329-335.

24. Hugonnet, J.-E., and Blanchard, J. S. (2007) Irreversible Inhibition of the Mycobacterium tuberculosis $\beta$ Lactamase by Clavulanate, Biochemistry 46, 11998-12004.

25. Wang, F., Cassidy, C., and Sacchettini, J. C. (2006) Crystal structure and activity studies of the Mycobacterium tuberculosis beta-lactamase reveal its critical role in resistance to beta-lactam antibiotics, Antimicrob. Agents Chemother. 50, 2762-2771.

26. Wang, F., Cassidy, C., and Sacchettini, J. C. (2006) Crystal Structure and Activity Studies of the Mycobacterium tuberculosis $\beta$-Lactamase Reveal Its Critical Role in Resistance to $\beta$-Lactam Antibiotics, Antimicrobial Agents and Chemotherapy 50, 2762-2771.

27. Tremblay, L. W., Fan, F., and Blanchard, J. S. (2010) Biochemical and Structural Characterization of Mycobacterium tuberculosis $\beta$-Lactamase with the Carbapenems Ertapenem and Doripenem, Biochemistry 49, 3766-3773.

28. Bassetti, M., Nicolini, L., Esposito, S., Righi, E., and Viscoli, C. (2009) Current status of newer carbapenems, Curr Med Chem 16, 564-575.

29. Hazra, S., Xu, H., and Blanchard, J. S. (2014) Tebipenem, a new carbapenem antibiotic, is a slow substrate that inhibits the beta-lactamase from Mycobacterium tuberculosis, Biochemistry 53, 3671-3678.

30. Dauby, N., Muylle, I., Mouchet, F., Sergysels, R., and Payen, M. C. (2011) Meropenem/clavulanate and linezolid treatment for extensively drug-resistant tuberculosis, Pediatr. Infect. Dis. J. 30, 812-813.

31. De Lorenzo, S., Alffenaar, J. W., Sotgiu, G., Centis, R., D'Ambrosio, L., Tiberi, S., Bolhuis, M. S., van Altena, R., Viggiani, P., Piana, A., Spanevello, A., and Migliori, G. B. (2013) Efficacy and safety of meropenem-clavulanate added to linezolid-containing regimens in the treatment of MDR-/XDR-TB, Eur. Respir. J. 41, 1386-1392.

32. Payen, M. C., De Wit, S., Martin, C., Sergysels, R., Muylle, I., Van Laethem, Y., and Clumeck, N. (2012) Clinical use of the meropenem-clavulanate combination for extensively drug-resistant tuberculosis, Int. J. Tuberc. Lung Dis. 16, 558-560.

33. England, K., Boshoff, H. I. M., Arora, K., Weiner, D., Dayao, E., Schimel, D., Via, L. E., and Barry, C. E. (2012) Meropenem-Clavulanic Acid Shows Activity against Mycobacterium tuberculosis In Vivo, Antimicrob. Agents Chemother. 56, 3384-3387.

34. Payen, M. C., Muylle, I., Vandenberg, O., Mathys, V., Delforge, M., Van den Wijngaert, S., Clumeck, N., and De Wit, S. (2018) Meropenem-clavulanate for drug-resistant tuberculosis: a follow-up of relapse-free cases, Int. J. Tuberc. Lung Dis. 22, 34-39.

35. Chambers, H. F., Kocagöz, T., Sipit, T., Turner, J., and Hopewell, P. C. (1998) Activity of Amoxicillin/Clavulanate in Patients with Tuberculosis, Clin. Infect. Dis. 26, 874-877.

36. Tiberi, S., Payen, M.-C., Sotgiu, G., D'Ambrosio, L., Alarcon Guizado, V., Alffenaar, J. W., Abdo Arbex, M., Caminero, J. A., Centis, R., De Lorenzo, S., Gaga, M., Gualano, G., Roby Arias, A. J., Scardigli, A., Skrahina, A., Solovic, I., Sulis, G., Tadolini, M., Akkerman, O. W., Alarcon Arrascue, E., Aleska, A., Avchinko, V., Bonini, E. H., Chong Marín, F. A., Collahuazo López, L., de Vries, G., Dore, S., Kunst, H., Matteelli, A., Moschos, C., Palmieri, F., Papavasileiou, A., Spanevello, A., Vargas Vasquez, D., Viggiani, P., White, V., Zumla, A., and Migliori, G. B. (2016) Effectiveness and safety of meropenem/clavulanatecontaining regimens in the treatment of MDR- and XDR-TB, Eur. Resp. J. 47, 1235-1243. 
37. Chambers, H. F., Turner, J., Schecter, G. F., Kawamura, M., and Hopewell, P. C. (2005) Imipenem for Treatment of Tuberculosis in Mice and Humans, Antimicrob. Agents Chemother. 49, 2816-2821.

38. Hugonnet, J.-E., Tremblay, L. W., Boshoff, H. I., Barry, C. E., and Blanchard, J. S. (2009) MeropenemClavulanate Is Effective Against Extensively Drug-Resistant Mycobacterium tuberculosis, Science 323, 1215-1218.

39. Solapure, S., Dinesh, N., Shandil, R., Ramachandran, V., Sharma, S., Bhattacharjee, D., Ganguly, S., Reddy, J., Ahuja, V., Panduga, V., Parab, M., Vishwas, K. G., Kumar, N., Balganesh, M., and Balasubramanian, V. (2013) In Vitro and In Vivo Efficacy of $\beta$-Lactams against Replicating and Slowly Growing/Nonreplicating Mycobacterium tuberculosis, Antimicrob. Agents Chemother. 57, 2506-2510.

40. Cho, S. H., Goodlett, D., and Franzblau, S. (2006) ICAT-based comparative proteomic analysis of nonreplicating persistent Mycobacterium tuberculosis, Tuberculosis 86, 445-460.

41. Puri, A. W., and Bogyo, M. (2009) Using Small Molecules To Dissect Mechanisms of Microbial Pathogenesis, ACS Chem. Biol. 4, 603-616.

42. Kocaoglu, O., and Carlson, E. E. (2016) Progress and prospects for small-molecule probes of bacterial imaging, Nat Chem Biol 12, 472-478.

43. Kolbe, K., Veleti, S. K., Johnson, E. E., Cho, Y.-W., Oh, S., and Barry, C. E. (2018) Role of Chemical Biology in Tuberculosis Drug Discovery and Diagnosis, ACS Infect. Dis.

44. Gee, K. R., Kang, H. C., Meier, T. I., Zhao, G., and Blaszcak, L. C. (2001) Fluorescent Bocillins: Synthesis and application in the detection of penicillin-binding proteins, Electrophoresis 22, 960-965.

45. June, C. M., Vaughan, R. M., Ulberg, L. S., Bonomo, R. A., Witucki, L. A., and Leonard, D. A. (2014) A fluorescent carbapenem for structure function studies of penicillin-binding proteins, $\beta$-lactamases, and $\beta$ lactam sensors, Anal. Biochem. 463, 70-74.

46. Kocaoglu, O., Calvo, R. A., Sham, L.-T., Cozy, L. M., Lanning, B. R., Francis, S., Winkler, M. E., Kearns, D. B., and Carlson, E. E. (2012) Selective Penicillin-Binding Protein Imaging Probes Reveal Substructure in Bacterial Cell Division, ACS Chem. Biol. 7, 1746-1753.

47. Kocaoglu, O., and Carlson, E. E. (2015) Profiling of $\beta$-Lactam Selectivity for Penicillin-Binding Proteins in Escherichia coli Strain DC2, Antimicrob. Agents Chemother. 59, 2785-2790.

48. Staub, I., and Sieber, S. A. (2008) $\beta$-Lactams as Selective Chemical Probes for the in Vivo Labeling of Bacterial Enzymes Involved in Cell Wall Biosynthesis, Antibiotic Resistance, and Virulence, J. Am. Chem. Soc. 130, 13400-13409.

49. Staub, I., and Sieber, S. A. (2009) $\beta$-Lactam Probes As Selective Chemical-Proteomic Tools for the Identification and Functional Characterization of Resistance Associated Enzymes in MRSA, J. Am. Chem. Soc. 131, 6271-6276.

50. The UniProt Consortium. (2017) UniProt: the universal protein knowledgebase, Nucleic Acids Res. 45, D158-D169.

51. Patru, M.-M., and Pavelka, M. S. (2010) A Role for the Class A Penicillin-Binding Protein PonA2 in the Survival of Mycobacterium smegmatis under Conditions of Nonreplication, J. Bacteriol. 192, 3043-3054.

52. Kieser, K. J., Boutte, C. C., Kester, J. C., Baer, C. E., Barczak, A. K., Meniche, X., Chao, M. C., Rego, E. H., Sassetti, C. M., Fortune, S. M., and Rubin, E. J. (2015) Phosphorylation of the Peptidoglycan Synthase PonA1 Governs the Rate of Polar Elongation in Mycobacteria, PLoS Pathog. 11, e1005010.

53. Sambandamurthy, V. K., Derrick, S. C., Jalapathy, K. V., Chen, B., Russell, R. G., Morris, S. L., and Jacobs, W. R. (2005) Long-Term Protection against Tuberculosis following Vaccination with a Severely Attenuated Double Lysine and Pantothenate Auxotroph of Mycobacterium tuberculosis, Infect. Immun. 73, 1196-1203.

54. Wayne, L. G. (2001) In Vitro Model of Hypoxically Induced Nonreplicating Persistence of Mycobacterium tuberculosis, Methods Mol. Med. 54, 247-269.

55. Tallman, K. R., Levine, S. R., and Beatty, K. E. (2016) Small Molecule Probes Reveal Esterases with Persistent Activity in Dormant and Reactivating Mycobacterium tuberculosis, ACS Infect. Dis. 9, 936-944.

56. Tallman, K. R., Levine, S. R., and Beatty, K. E. (2016) Profiling Esterases in Mycobacterium tuberculosis Using Far-Red Fluorogenic Substrates, ACS Chem. Biol. 11, 1810-1815.

57. Rezwan, M., Lanéelle, M.-A., Sander, P., and Daffé, M. (2007) Breaking down the wall: Fractionation of mycobacteria, Journal of Microbiological Methods 68, 32-39.

58. Flores, A. R., Parsons, L. M., and Pavelka, Martin S. (2005) Genetic analysis of the $\beta$-lactamases of Mycobacterium tuberculosis and Mycobacterium smegmatis and susceptibility to $\beta$-lactam antibiotics, Microbiology 151, 521-532. 
59. Tremblay, L. W., Hugonnet, J. E., and Blanchard, J. S. (2008) Structure of the covalent adduct formed between Mycobacterium tuberculosis beta-lactamase and clavulanate, Biochemistry 47, 5312-5316.

60. Prisic, S., Dankwa, S., Schwartz, D., Chou, M. F., Locasale, J. W., Kang, C.-M., Bemis, G., Church, G. M., Steen, H., and Husson, R. N. (2010) Extensive phosphorylation with overlapping specificity by Mycobacterium tuberculosis serine/threonine protein kinases, Proceedings of the National Academy of Sciences 107, 7521-7526.

61. de Souza, G. A., Leversen, N. A., Målen, H., and Wiker, H. G. (2011) Bacterial proteins with cleaved or uncleaved signal peptides of the general secretory pathway, J. Proteomics 75, 502-510.

62. Bacon, J., Alderwick, L. J., Allnutt, J. A., Gabasova, E., Watson, R., Hatch, K. A., Clark, S. O., Jeeves, R. E., Marriott, A., Rayner, E., Tolley, H., Pearson, G., Hall, G., Besra, G. S., Wernisch, L., Williams, A., and Marsh, P. D. (2014) Non-Replicating Mycobacterium tuberculosis Elicits a Reduced Infectivity Profile with Corresponding Modifications to the Cell Wall and Extracellular Matrix, PLOS ONE 9, e87329.

63. Seiler, P., Ulrichs, T., Bandermann, S., Pradl, L., Jörg, S., Krenn, V., Morawietz, L., Kaufmann, S. H. E., and Aichele, P. (2003) Cell-Wall Alterations as an Attribute of Mycobacterium tuberculosis in Latent Infection, J. Infect. Disease 188, 1326-1331. 